\title{
Serum albumin level adjusted with C- reactive protein predicts hemodialysis patient survival
}

Norio Hanafusa ${ }^{1}$, Kosaku Nitta ${ }^{2 *}$, Masayuki Okazaki ${ }^{3}$, Mizuki Komatsu ${ }^{3}$, Shunji Shiohira ${ }^{2,3}$, Hiroshi Kawaguchi ${ }^{3}$ and Ken Tsuchiya ${ }^{1}$

\begin{abstract}
Background: Malnutrition-related conditions such as protein energy wasting are affecting poor outcome of the dialysis population. The serum albumin level is often used as a marker of wasting and malnutrition. However, the serum albumin level can be affected by the presence of inflammation as well as by nutritional status. We investigated associations between serum albumin values adjusted with C-reactive protein (CRP) and mortality in hemodialysis (HD) patients.

Methods: A total of $397 \mathrm{HD}$ patients were included in this study. The patient characteristics and laboratory data, including serum albumin and CRP levels, were obtained in July 2012. Survival as of the end of August 2014 (maximum follow-up period 25 months) was investigated. First, the regression line was obtained from the entire population between serum albumin and CRP levels. Next, the CRP-adjusted albumin levels were determined as a dichotomized variable with the patients having higher albumin values above the regression line. We investigated associations between three albumin indices (the actual values, the dichotomized index by median, and (RP-adjusted values) and mortality by using Cox proportional hazard models.

Results: The average age of the 397 HD patients was 70.6 years old; 258 were male, and $44.6 \%$ were diabetic. Median dialysis vintage was 3.6 years. There were 73 deaths during the observation period. A univariate Cox analysis demonstrated that all three indices were associated with survival, and the hazard ratio (HR) was 0.40 (95\% confidence interval (Cl) 0.25-0.68) for increase in albumin level, 0.41 (95\% Cl 0.25-0.68) for higher dichotomized albumin level by median, and 0.46 (95\% Cl 0.28-0.74) for higher CRP-adjusted albumin level, but only higher CRPadjusted albumin level was associated with better survival (HR 0.51,95\% Cl 0.30-0.85). The degree to which the deviation of albumin was attributable to that of CRP was $12.6 \%$. The analyses on cause-specific mortality revealed that lower albumin levels were associated significantly with infection-related mortality but were not significantly associated with death due to congestive heart failure.
\end{abstract}

Conclusions: CRP-adjusted albumin was shown to be a better predictor of mortality among the HD patients. When assessing serum albumin values, inflammatory status should be taken into account. Malnutrition or wasting was shown to be associated with a poor outcome independent of inflammation.

Keywords: Albumin, C-reactive protein, Cohort study, Hemodialysis, Mortality, Protein energy wasting

\footnotetext{
* Correspondence: knitta@kc.twmu.ac.jp

${ }^{2}$ Department of Medicine, Kidney Center, Tokyo Women's Medical University,

8-1 Kawada-cho, Shinjuku-ku, Tokyo 162-8666, Japan

Full list of author information is available at the end of the article
} International License (http://creativecommons.org/licenses/by/4.0/), which permits unrestricted use, distribution, and reproduction in any medium, provided you give appropriate credit to the original author(s) and the source, provide a link to the Creative Commons license, and indicate if changes were made. The Creative Commons Public Domain Dedication waiver (http://creativecommons.org/publicdomain/zero/1.0/) applies to the data made available in this article, unless otherwise stated. 


\section{Background}

The dialysis population in Japan is becoming older. Malnutrition-related conditions such as protein energy wasting (PEW) [1-4], sarcopenia [5, 6], and frailty [4, 7] draw attentions among such older dialysis populations. Many studies have investigated the relationship between malnutrition and patient outcome, and the results have revealed that malnutrition-related conditions are associated with a poor outcome [5-7].

To investigate the best index that reflects malnutrition is a matter of debate [8-12]. The serum albumin level is one of the most popular indexes of malnutrition $[2,8,11$, 12]. Albumin reportedly relates to the poor survival in original values [13], together with in a form of geriatric nutritional risk index (GNRI) [11]. However, starvation does not necessarily reduce albumin values [14], and the inflammatory state that often coincides with malnutrition in dialysis populations [1] largely affects the lower albumin values [14]. Thus, the serum albumin level might not be a suitable marker of malnutrition in dialysis patients.

We speculated that the values that deducted the effect of inflammation from the actual albumin might become a better marker of malnutrition compared to the original albumin values independent of the effect of inflammation. We therefore performed a study to investigate the relationship between the serum albumin levels and $\mathrm{C}$ reactive protein (CRP) levels, and thereafter, the albumin values adjusted with CRP were investigated from the perspective of all-cause mortality of hemodialysis (HD) patients.

\section{Methods}

\section{Design and population}

This was a retrospective, observational cohort study conducted at a single center in Japan. The potential population was the chronic dialysis population that was on HD therapy through an arteriovenous fistula at Jyoban Hospital, Iwaki, Japan, in July 2012. HD patients with a malignancy, active inflammation, liver cirrhosis, gastrointestinal bleeding, cardiac valvular disease, or severe illness were excluded from participation and were transferred to another dialysis unit for intensive care. We also excluded the data of patients who did not consent to participate in this study. The Institutional Review Board of the Jyoban Hospital approved the study protocol; the approval number is 25-(3). The protocols were carried out in accordance with the Declaration of Helsinki guidelines regarding ethical principles for medical research involving human subjects. Informed consent was obtained from every subject.

\section{Laboratory measurements}

The laboratory data were obtained on the basis of daily clinical practice. The biochemistry data and complete blood counts were obtained on the first day of each week. All measurements were made except the blood urea nitrogen (BUN) level before a dialysis session. BUN level was measured both before and after the dialysis session. Serum creatinine, calcium, phosphorus, albumin, total cholesterol, high-density lipoprotein (HDL) cholesterol, and triglyceride were measured with an autoanalyzer by standard laboratory methods. The serum calcium values were corrected with albumin level. Intact parathyroid hormone (iPTH) was measured by an immunoradiometric assay. Body mass index (BMI) was expressed in $\mathrm{kg} / \mathrm{m}^{2}$. Body weight was calculated as dry weight, defined as post-dialysis weight in which the patient was normotensive and with no signs of overhydration. Urea kinetics were assessed by measuring a bloodbased dialysis parameter, Kt/V [15], and the mean value of the three measurements during each of the 3 months before the start of the study was used in the analysis. The normalized protein catabolic rate (nPCR) was used as an indirect indicator of protein intake and was calculated by using the formula previously reported [16].

The history of cardiovascular disease was defined from medical records showing previous heart failure, myocardial infarction, angina pectoris, stroke, coronary artery disease combined with stent implantation, and peripheral artery disease combined with percutaneous transluminal angioplasty.

\section{Clinical outcomes}

Endpoints were obtained from the hospital charts and by telephone interview with patients that were conducted by trained interviewers who were blinded to the study protocol. The primary endpoint of this study was all-cause mortality during the follow-up period, from July 1, 2012 to August 31, 2014. The vital status of the subjects was assessed by searching their electronic dialysis records. Patients were censored if the patient was alive on August 31, 2014.

\section{Statistical analyses}

For the descriptive analysis, the mean values and standard deviations or median and interquartile ranges (IQRs) were used for normally or non-normally distributed continuous variables. Categorical variables were summarized by numbers and proportion.

The relationship between serum albumin and CRP levels was determined. Linear regression analysis was performed, and the predictive equation of albumin adjusted with CRP was determined. CRP is known to have a log-transformed normal distribution, and log CRP values were used to evaluate the relationship between the CRP and albumin. From the equation, the predicted albumin values that were adjusted with CRP levels were determined. 
We performed the survival analysis by using the albumin indices as the main predictors. We used albumin indices in three ways: actual values, albumin dichotomized by the median, and CRP-adjusted albumin that was determined as high or low values, if the actual albumin values were higher or lower than predicted by the CRP levels. We performed Kaplan-Meier analyses followed by log-rank tests for the dichotomized albumin values by the median and CRP-adjusted albumin. Next, a Cox proportional hazard model was employed to evaluate survival effects of albumin. First, we performed univariate analyses, and then the models were adjusted for age, gender, diabetes, dialysis vintage, nPCR, and $\log C R P$. Dialysis vintages were divided into four groups $(<2$ years, $2-4$ years, $4-8$ years, $\geq 8$ years). Because the median dialysis vintage was 3.6 years and IQR was $1.6-7.5$, such limits yielded almost quartilized groups in terms of the length of dialysis vintage. We used <2-year dialysis vintage group as the reference group. The effects of variables are expressed in the form of hazard ratios (HRs) and $95 \%$ confidence intervals (CIs).

We also performed similar survival analyses for the mortalities due to congestive heart failure and infective diseases. The causes of death were collected as the forms of semi-categorized disease entities. The deaths due to congestive heart failure were defined as the death due to itself, while the deaths due to infection included sepsis, pneumonia, and acute cholecystitis. The numbers of the deaths due to either congestive heart failure or infection were limited. Thus, in the multivariate analyses to investigate the associations of each albumin index with the cause-specific mortalities, we only adjusted age, gender, and diabetes as primary diagnoses. Actually, the numbers of deaths due to infection were small, and there remains a concern that we included more variables to adjust in the models. The degree of model fittings was also evaluated by the corrected Akaike's Information Criteria (AICc). Furthermore, in order to investigate more detailed association, we also made a cross table for the numbers of the deceased patients by dichotomized albumin values and CRP-adjusted albumin values. The significance of this association was investigated by Fisher's exact test.

The JMP 11.2.0 software program (SAS Institute Inc., NC, USA) was used to perform the statistical analysis.

\section{Results}

Patient characteristics are shown in Table 1. The average age of the patients was 70.6 years old, which was slightly higher than the average age of total HD population of Japan (67.2 \pm 12.5 years old at the end of 2013) [15]. Diabetes and nephrosclerosis were the predominant primary diagnoses. One fourth of the patients had a history of cardiovascular disease.
Table 1 Baseline background factors of the subjects

\begin{tabular}{ll}
\hline Items & Values \\
\hline Age (years) & $70.6 \pm 13.4$ (median 72, IQR 62-81) \\
Gender (female/male) & $139 / 258$ \\
Primary diagnosis (person, \%) & $177(44.6 \%)$ \\
$\quad$ Diabetes & $94(23.7 \%)$ \\
$\quad$ Nephrosclerosis & $76(19.1 \%)$ \\
Glomerulonephritis & $50(12.6 \%)$ \\
Others & Median 3.6, IQR 1.6-7.5 \\
Dialysis vintage (years) & $103(25.9 \%)$ \\
History of cardiovascular & \\
diseases (person, \%) & $152(43.3 \%$, unknown excluded \\
History of smoking (persons, \%) & from the population) \\
Physical indices & \\
Body height (cm) & $159.3 \pm 9.9$ \\
Dry weight (kg) & $56.6 \pm 12.2$ \\
Body mass index (kg/m²) & $22.1 \pm 3.8$ \\
\hline
\end{tabular}

The laboratory data are summarized in Table 2. The mean serum albumin value was $3.62 \pm 0.38 \mathrm{~g} / \mathrm{dl}$ (median 3.7, IQR 3.4-3.9). The median serum CRP value was $0.14 \mathrm{mg} / \mathrm{dl}$, and the IQR was $0.05-0.38 \mathrm{mg} / \mathrm{dl}$, which can be rephrased as $\log \mathrm{CRP}-0.84 \pm 0.62(\log \mathrm{mg} / \mathrm{dl})$.

Figure 1 shows the relationship between the $\log C R P$ and albumin values, and a weak but significant correlation was found between the two sets of values. The regression equation was albumin $(\mathrm{g} / \mathrm{dl})=3.438-0.215$ $\log$ CRP $(\log \mathrm{mg} / \mathrm{dl})$. The degree of fitting was expressed as $R^{2}=0.126$, which means that $12.6 \%$ of the albumin distribution was determined by the $\log C R P$ value.

The numbers of patients who died during the study period and their causes of death are summarized in Table 3. In total, 73 patients had died from all causes. The most common cause of death was heart failure $(42.5 \%)$, and it was followed by infection $(23.3 \%)$ and cerebrovascular disease (11.0\%).

Kaplan-Meier analyses followed by log-rank tests demonstrated that both the higher dichotomized and CRP-adjusted albumin level groups were significantly associated with better survival (Fig. 2a, b). The univariate analysis was performed to investigate the relationships between each value and the all-cause mortality, and significant associations were found with age, log CRP, and albumin (Table 4). The relationship between serum albumin levels and mortality was investigated in three ways, as described above. The first was the actual value, the second was the dichotomized value, and the last was the albumin values adjusted with CRP. The CRP-adjusted albumin values were the dichotomized value that was determined by the albumin predicted from CRP values with the use of the regression equation, i.e., predicted 
Table 2 Baseline laboratory data of the subjects

\begin{tabular}{ll}
\hline Items & Measurements \\
\hline Systolic blood pressure (mmHg) & $153.5 \pm 17.6$ \\
Diastolic blood pressure (mmHg) & $80.0 \pm 12.1$ \\
Pulse rate (per minute) & $73.5 \pm 13.4$ \\
Albumin (g/dl) & $3.62 \pm 0.38$ (median 3.7, IQR 3.4-3.9) \\
CRP (mg/dl) & Median 0.14, IQR 0.05-0.38 \\
& (range 0.01-15.54) \\
LogCRP (log mg/dl) & $-0.84 \pm 0.62$ (median -0.85, \\
& IQR -1.30 to -0.42) \\
Hemoglobin (g/dl) & $10.5 \pm 1.3$ \\
Ferritin (ng/ml) & Median 60, IQR 23-123 \\
Transferrin saturation (\%) & $24.7 \pm 13.2$ \\
Total cholesterol (mg/dl) & $152.7 \pm 33.0$ \\
HDL cholesterol (mg/dl) & $46.2 \pm 13.8$ \\
Triglyceride (mg/dl) & $113.1 \pm 71.7$ (median 94, IQR 64-138) \\
LDL cholesterol (mg/dl) & $83.8 \pm 28.0$ \\
Albumin-corrected calcium (mg/dl) & $9.08 \pm 0.65$ \\
Phosphate (mg/dl) & $4.67 \pm 1.75$ \\
Intact PTH (pg/ml) & Median 101, IQR 54-179 \\
Kt/N & $1.34 \pm 0.27$ \\
nPCR (g/kg/day) & $0.83 \pm 0.19$ \\
\hline CRPC-rear &
\end{tabular}

CRP C-reactive protein, HDL high-density lipoprotein, LDL low-density lipoprotein, $P T H$ parathyroid hormone, and $n P C R$ normalized protein catabolic rate albumin $(\mathrm{g} / \mathrm{dl})=3.438-0.215 \log \mathrm{CRP}(\log \mathrm{mg} / \mathrm{dl})$. If the predicted values were smaller than the actual values, the CRP-adjusted albumin values were determined as low, vice versa. All three indices were significantly negatively correlated with all-cause mortality.

We investigated the predictive power of three albumin indices adjusted by age, gender, diabetes, dialysis vintage, nPCR, and $\log C R P$. The HR for these indices was 0.60 (95\% CI 0.29-1.23) for the actual serum albumin values, 0.64 (95\% CI $0.37-1.08$ ) for the dichotomized albumin values, and 0.51 (95\% CI 0.30-0.85) for the CRPadjusted albumin values. As a result, only CRP-adjusted albumin value was significantly associated with the poorer outcome (Table 5).

For cause-specific analyses, the lower groups for the albumin indices tended to be associated with lower mortality due to congestive heart failure but did not reach their significances by crude models. The HRs (and 95\% CIs) for albumin indices were 0.44 (0.21-1.03), 0.52 (0.24-1.08), and $0.67(0.32-1.37)$, for actual albumin values, dichotomized albumin values by the median, and CRP-adjusted albumin values, respectively. This association did not change even after adjustment of age, gender, and diabetes as primary diagnosis (Table 6). AICc is an index of the degree of model fitting, and the lower value demonstrates better model fitting. AICc demonstrated that

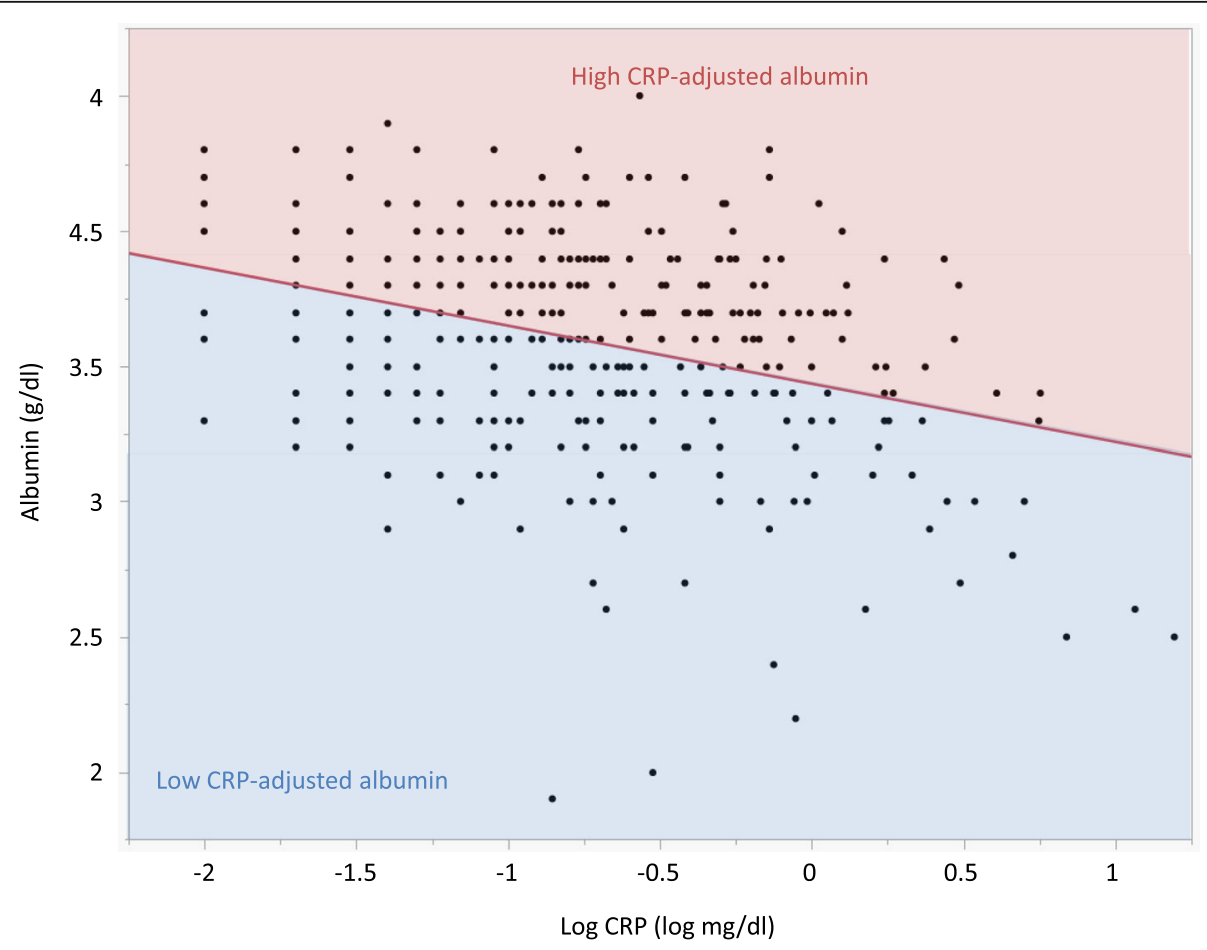

Fig. 1 Relationship between log CRP values and serum albumin values. A scatter plot of log CRP values and serum albumin values is shown. The regression line was obtained by the least squares method. The albumin values above the regression line were considered high CRP-adjusted albumin values, and the values below the regression line low CRP-adjusted serum albumin values 
Table 3 Causes of death

\begin{tabular}{ll}
\hline Causes for death & Numbers (\%) \\
\hline All causes & 73 \\
Cause-specific & \\
Heart failure & $31(42.5 \%)$ \\
Infection & $17(23.3 \%)$ \\
Cerebrovascular & $8(11.0 \%)$ \\
Malignancy & $5(6.8 \%)$ \\
Myocardial infarction & $5(6.8 \%)$ \\
Gastrointestinal bleeding & $4(5.5 \%)$ \\
Other causes & $3(4.1 \%)$ \\
\hline
\end{tabular}

CRP-adjusted albumin did not improve the degree of model fitting compared to other indices.

On the other hand, the associations between albumin indices and death due to infection were significant for all of the indices investigated by crude models. The HRs (and 95\% CIs) for albumin indices were 0.22 (0.09-0.58), $0.12(0.02-0.42)$, and $0.12(0.02-0.41)$, for actual albumin values, dichotomized albumin values by the median, and CRP-adjusted albumin values, respectively. The associations did not change their significances. However, the CRP-adjusted albumin levels demonstrated the lower HR compared to that of the dichotomized albumin by the median. AICc of the model which included CRPadjusted albumin was also the lowest among the three models, which indicates the model exhibited the best fitting (Table 7).

Table 8 indicated that the patients in the group with lower dichotomized by the median and lower CRPadjusted albumin levels demonstrated higher infectionrelated mortality $(p<0.001)$, while the patients in the group with higher dichotomized by the median and higher CRP-adjusted levels demonstrated better survival $(p=0.005)$.

\section{Discussion}

The results of this study showed that $12.6 \%$ of the deviation of albumin was determined by CRP. The CRPadjusted albumin value was the only index associated with a poorer outcome of the chronic HD patients in this study. This result means that malnutrition or wasting itself was associated with survival, independent of the inflammation status, or protein intake of the chronic HD patients in this study. The analyses for causespecific death demonstrated that such association was evident for death due to infection but not for congestive heart failure.

PEW is a condition in which visceral protein and fat stores decrease as a result of many chronic comorbid conditions, including chronic kidney disease $[1,2]$. Since PEW has been shown to be associated with a poor outcome in many clinical investigations [1, 3, 4], strategies to combat PEW have been anticipated and discussed [1, 3]. The International Society of Renal Nutrition and Metabolism published diagnostic criteria for PEW [2]. The guideline employed four clinical indices: serum chemistry (low serum levels of albumin, prealbumin, and cholesterol), body mass (reduced BMI, weight loss, and reduction of total body fat), muscle mass (muscle wasting, anthropometry, and creatinine appearance), and dietary intake (low dietary protein intake and energy intake) [2]. Thus, reduced serum albumin level is closely related to PEW. Actually, the population with even minimal reduction of albumin as well as low albumin values reportedly experiences poor survival [13]. The results from Kaplan-Meier analyses of this study confirmed such effects of albumin on mortality and demonstrated that lower albumin levels related to worse outcome.

Albumin is synthesized in the liver, and albumin synthesis is influenced by inflammation status [16] and amino acid pools $[17,18]$. Moreover, inflammatory status shortens the half-life of serum albumin [19]. Serum albumin level is therefore considered as an index of both inflammation and wasting or malnutrition $[1,14]$. However, since dietary restriction does not always lead to a reduction in serum albumin level [14], the significance of inflammation has been postulated and been shown to outweigh the effect of malnutrition in determining the serum albumin levels [14]. Likewise, the reduction of the serum albumin level is not necessarily caused by the malnutrition itself [14]. An experiment in 1944 to 1945 investigated the effect of starvation on the physiological and psychological aspects of healthy volunteers. The results showed that only a limited reduction in serum albumin level (from $4.3 \pm 0.5$ to $3.9 \pm 0.5 \mathrm{~g} / \mathrm{dl}$ ) was observed even after a severe decrease in body mass (BMI: from $21.7 \pm 1.7$ to $16.4 \pm 0.9 \mathrm{~kg} / \mathrm{m}^{2}$ ) [14]. Moreover, the inflammation-related conditions or wasting are considered to have larger impacts on the reduction of albumin levels [3]. Actually, the results of the present study indicated that $12.6 \%$ of deviation in albumin values was derived from those of CRP. Therefore, the degree was not so large, but it was demonstrated that albumin could be influenced by inflammation.

HD patients are prone to have inflammatory status because of the vascular access [20], periodontal diseases [21], bacterial translocation from the altered microbiome in the gut [22], or immunodeficiency [23]. The presence of inflammation can be related to worse survival among HD patients [24, 25]; the HD patients with higher serum CRP level have higher mortality rates [25]. However, the serum CRP levels of our study population were no longer associated with the poor outcome after adjustment for the serum albumin levels. This finding indicated that 
a

Survival Rate

Higher Dichotomized Albumin Level

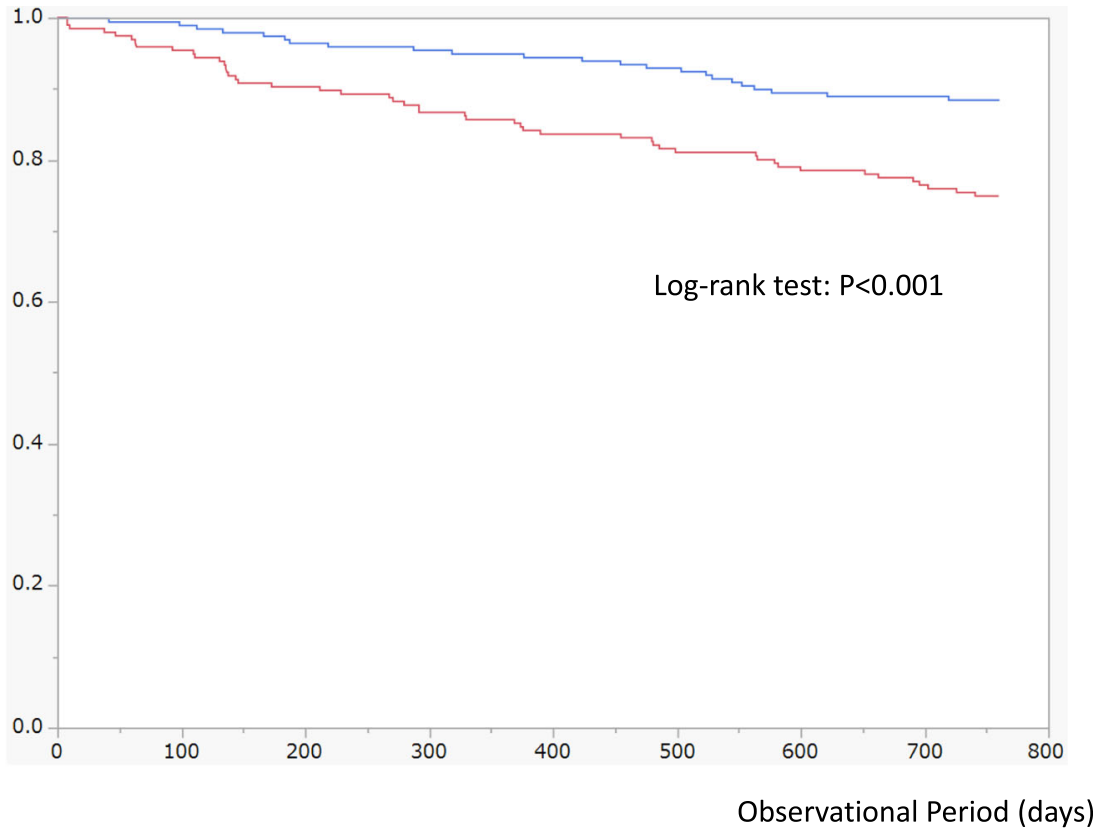

b

Survival Rate

Higher CRP-adjusted Albumin Level

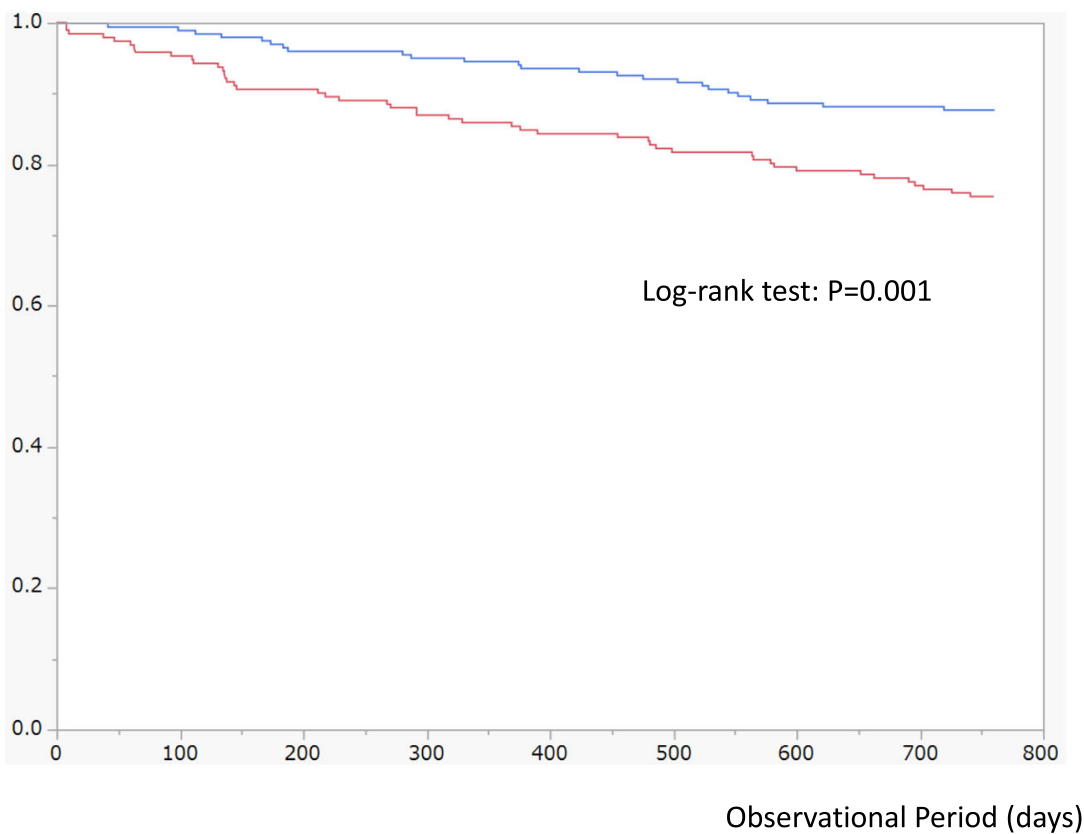

Fig. 2 The survivals of the groups with the higher and lower albumin indices. The Kaplan-Meier analyses and log-rank tests were performed for the dichotomized (a) or CRP-adjusted (b) albumin values. The blue lines indicate the higher group, and the red lines indicate the lower group. The higher groups for both indices exhibited the better survival compared to the lower groups

the significance of wasting or malnutrition might have larger impact on the better health of HD patients compared to inflammation.
This study demonstrated that such association was most evident for death due to infection. A study investigated the effect of albumin, which was not adjusted for 
Table 4 Univariate analysis of the associations between clinical indices and all-cause mortality

\begin{tabular}{ll}
\hline Parameters & $\mathrm{HR}(95 \% \mathrm{Cl})$ \\
\hline Age (per 1 year) & $1.07(1.05-1.09)$ \\
Gender (female) & $0.71(0.42-1.17)$ \\
Diabetes & $1.53(0.96-2.44)$ \\
Dialysis vintage (years) & \\
$\quad<2$ & Reference \\
$\geq 2$ and $<4$ & $0.85(0.45-1.59)$ \\
$\geq 4$ and $<8$ & $0.84(0.44-1.54)$ \\
$\geq 8$ & $0.76(0.36-1.44)$ \\
nPCR (per 1 g/kg/day) & $0.61(0.18-2.02)$ \\
LogCRP (per 1 log mg/dl) & $1.79(1.25-2.54)$ \\
Actual albumin (per 1 g/dl) & $0.40(0.25-0.68)$ \\
Dichotomized albumin by median (the higher group) & $0.41(0.25-0.68)$ \\
CRP-adjusted albumin (the higher group) & $0.46(0.28-0.74)$ \\
\hline
\end{tabular}

nPCR normalized protein catabolic rate, CRP C-reactive protein, $H R$ hazard ratio, and $\mathrm{Cl}$ confidence intervals

CRP, on cause-specific mortality across hemodialysis and peritoneal dialysis [26]. The study found that the HRs of low albumin levels for infection-related mortality were higher than those for cardiovascular mortality irrespective of dialysis modalities. Our study confirmed this result even by CRP-adjusted albumin. Moreover, the group with the lower CRP-adjusted albumin values more

Table 5 Predictive power of each of the different albumin indices

\begin{tabular}{llll}
\hline Parameters & Model 1 & Model 2 & Model 3 \\
\hline Age (per 1 year) & $1.08(1.05-1.11)$ & $1.08(1.05-1.11)$ & $1.08(1.05-1.10)$ \\
Gender (female) & $0.50(0.28-0.87)$ & $0.49(0.27-0.84)$ & $0.50(0.28-0.86)$ \\
Diabetes & $1.82(1.12-2.96)$ & $1.77(1.09-2.88)$ & $1.84(1.14-3.00)$
\end{tabular}

Dialysis vintage (years)

\begin{tabular}{llll}
$\quad<2$ & Reference & Reference & Reference \\
$\geq 2$ and $<4$ & $0.84(0.42-1.66)$ & $0.78(0.40-1.47)$ & $0.89(0.45-1.70)$ \\
$\geq 4$ and $<8$ & $0.90(0.46-1.72)$ & $0.84(0.44-1.56)$ & $0.92(0.48-1.73)$ \\
$\geq 8$ & $1.35(0.65-2.71)$ & $1.31(0.64-2.57)$ & $1.39(0.68-2.70)$ \\
$\mathrm{nPCR}$ (per $1 \mathrm{~g} /$ & $2.30(0.64-7.90)$ & $2.16(0.62-7.29)$ & $2.28(0.66-7.55)$ \\
$\mathrm{kg} / \mathrm{day})$ & & & \\
$\begin{array}{l}\text { Log CRP (per 1 } \\
\text { log mg/dl) }\end{array}$ & $1.21(0.81-1.80)$ & $1.24(0.84-1.82)$ & $1.43(0.97-2.10)$ \\
$\begin{array}{l}\text { Actual albumin } \\
\text { (per } 1 \text { g/dl) }\end{array}$ & $0.60(0.29-1.23)$ & - & - \\
$\begin{array}{l}\text { Dichotomized } \\
\text { albumin by median } \\
\text { (the higher group) }\end{array}$ & - & $0.64(0.37-1.08)$ & - \\
$\begin{array}{l}\text { CRP-adjusted } \\
\text { albumin } \\
\text { (the higher group) }\end{array}$ & - & & \\
\hline
\end{tabular}

nPCR normalized protein catabolic rate, CRP C-reactive protein, $H R$ hazard ratio, and $\mathrm{Cl}$ confidence interval
Table 6 Predictive power of each of the different albumin indices on mortality due to congestive heart failure

\begin{tabular}{llll}
\hline Parameters & Model 1 & Model 2 & Model 3 \\
\hline Age (per 1 year) & $1.09(1.05-1.13)$ & $1.09(1.05-1.14)$ & $1.09(1.05-1.13)$ \\
Gender (female) & $0.57(0.25-1.23)$ & $0.56(0.24-1.19)$ & $0.55(0.24-1.19)$ \\
Diabetes & $1.65(0.79-3.46)$ & $1.64(0.78-3.44)$ & $1.66(0.80-3.47)$ \\
$\begin{array}{l}\text { Actual albumin } \\
\text { (per } 1 \mathrm{~g} / \mathrm{dl} \text { ) }\end{array}$ & $0.71(0.27-1.89)$ & - & - \\
$\begin{array}{l}\text { Dichotomized } \\
\text { albumin by median } \\
\text { (the higher group) }\end{array}$ & - & $0.85(0.38-1.82)$ & - \\
$\begin{array}{l}\text { CRP-adjusted albumin } \\
\text { (the higher group) }\end{array}$ & & & \\
$\begin{array}{l}\text { Corrected Akaike's } \\
\text { Information Criteria }\end{array}$ & 335.1 & - & $0.87(0.41-1.80)$ \\
$\begin{array}{l}\text { CRP C-reactive protein } \\
\text { CR }\end{array}$ & 335.4 & 335.4 \\
\end{tabular}

significantly associated, which was shown by the lower AICc score, with higher infection-related mortality compared to albumin indices. The fact indicated that lower albumin without the effect of inflammatory status was more likely associated with the death due to infection. Further studies are required to investigate whether hypoalbuminemia itself [13] or malnutrition existing in the background [27] is associated with infectionrelated mortality.

On the other hand, albumin values were not significantly associated with survival for the death due to congestive heart failure, though the point estimates of HRs favored the higher albumin values for all indices. This fact indicates that low albumin values can relate to the death due to congestive heart failure, but other factors seemed more likely to be associated with such mortality. Moreover, interestingly enough, CRP-adjusted albumin levels were no longer significant compared to other albumin indices. The previous reports demonstrated that

Table 7 Predictive power of each of the different albumin indices on mortality due to infection

\begin{tabular}{llll}
\hline Parameters & Model 1 & Model 2 & Model 3 \\
\hline Age (per 1 year) & $1.15(1.08-1.22)$ & $1.14(1.07-1.21)$ & $1.14(1.07-1.21)$ \\
Gender (female) & $0.60(0.20-1.66)$ & $0.53(0.18-1.44)$ & $0.49(0.16-1.34)$ \\
Diabetes & $4.21(1.52-13.5)$ & $3.81(1.38-12.2)$ & $4.04(1.46-13.0)$ \\
$\begin{array}{l}\text { Actual albumin } \\
\text { (per 1 g/dl) }\end{array}$ & $0.24(0.07-0.93)$ & - & - \\
$\begin{array}{l}\text { Dichotomized } \\
\text { albumin by median } \\
\text { (the higher group) }\end{array}$ & - & $0.25(0.04-0.93)$ & - \\
$\begin{array}{l}\text { CRP-adjusted albumin } \\
\text { (the higher group) }\end{array}$ & - & & $0.17(0.03-0.62)$ \\
$\begin{array}{l}\text { Corrected Akaike's } \\
\text { Information Criteria }\end{array}$ & 169.4 & - & 165.9 \\
\hline
\end{tabular}

$n P C R$ normalized protein catabolic rate, CRP C-reactive protein, $H R$ hazard ratio, and $\mathrm{Cl}$ confidence interval 
Table 8 Distribution of the numbers of deceased patients. Predictive power of each of the different albumin indices on mortality due to infection

\begin{tabular}{llll}
\hline & & \multicolumn{2}{c}{ CRP-adjusted albumin } \\
\cline { 3 - 4 } & & The higher group & The lower group \\
\hline $\begin{array}{l}\text { Dichotomized } \\
\text { albumin } \\
\text { by median }\end{array}$ & $\begin{array}{l}\text { The higher } \\
\text { group }\end{array}$ & $2 / 180(1.11 \%)$ & $0 / 21(0 \%)$ \\
& $\begin{array}{l}\text { The lower } \\
\text { group }\end{array}$ & $0 / 25(0 \%)$ & $15 / 170(8.82 \%)$ \\
\hline
\end{tabular}

hypoalbuminemia can relate to cardiovascular diseases [28, 29]. However, it has been demonstrated that there are many other cardiovascular risk factors, and the condition is known as cardiorenal syndrome type 4 [30]. The present study confirmed that the death due to congestive heart failure might be associated with hypoalbuminemia itself, but more substantially with other risk factors as well. These associations require further investigations among larger cohorts.

Several indices, including the malnutrition-inflammation score [8] or GNRI [12], have been proposed to monitor the nutritional status of HD populations, and they have been reported to be good predictors of patient outcome $[8,11]$. However, from a clinical standpoint, serum albumin levels can be monitored easily and are widely used to assess global nutritional status. The results of the present study demonstrated that it is important to consider inflammatory status of HD patients when assessing their serum albumin levels. This finding also indicates that malnutrition or wasting can affect the outcome of the patients independent of their inflammation status, thereby confirming the value of nutritional interventions to combat malnutrition in HD populations [1].

\section{Limitations}

This study had several limitations. The first limitation was that it was performed on the basis of observational study, and thus, the cause result relationship cannot be drawn whether the outcome can be improved by the increase of serum albumin levels in face of higher CRP or the reduction of CRP in face of lower albumin levels. Second, this study was performed on the database of a single center. Further generalizability of the present investigation will be required by the study using the data of other cohorts. The final limitation was that only the baseline data were analyzed, and no changes in clinical indices, including albumin or CRP values, were considered. Substantial changes in the clinical parameters could take place afterward during the course. However, this study had several advantages. First, the total number of patients was substantial, and we were able to obtain a conclusion from this HD population. Second, the follow-up period and the numbers of outcomes are also sufficient to be investigated. Third, since the patient background factors almost matched those of the entire Japanese HD population. Therefore, the cohort in our study was the representative of the Japanese HD population.

\section{Conclusions}

When assessing the serum albumin levels of HD population, the inflammation status of the patients should be taken into consideration. Wasting or malnutrition itself can predict a worse outcome independent of inflammation.

\section{Abbreviations}

BMI: Body mass index; CRP: C-reactive protein; GNRI: Geriatric nutritional risk index; HDL: High-density lipoprotein; HR: Hazard ratio; IQR: Interquartile range; LDL: Low-density lipoprotein; $\mathrm{nPCR}$ : Normalized protein catabolic rate; PEW: Protein energy wasting; PTH: Parathyroid hormone

\section{Acknowledgements \\ Authors acknowledge all the participants and medical staffs who were engaged in the present investigations.}

\section{Funding}

The authors did not have specific funding source to be disclosed.

\section{Availability of data and materials}

The cohort data used in this article contains anonymized but individual data. Therefore, we prefer not to share this database.

\section{Authors' contributions}

$\mathrm{NH}$ performed the statistical analysis and wrote the draft version of this article. KN and KT planned, organized, and directed the investigation, as well as finalized the manuscript. MO, MK, SS, and HK were in charge of the investigated patients, collected clinical data, and finalizing and reviewing the manuscript. All authors read and approved the final manuscript.

\section{Competing interests}

$\mathrm{NH}$ was a former member of the division funded by the Terumo Corporation. $\mathrm{NH}$ had a research contract concerning oral nutritional support on dialysis patients with Meiji Co., Ltd.

\section{Consent for publication}

This article does not contain any individual personal information. Therefore, this section is not applicable.

\section{Ethics approval and consent to participate}

The Institutional Review Board of the Jyoban Hospital approved the study protocol (no. 25-(3)), and the protocols were carried out in accordance with the Declaration of Helsinki guidelines regarding ethical principles for medical research involving human subjects. Informed consent was obtained from every subject. The written informed consent was obtained from the patients.

\section{Author details}

'Department of Blood Purification, Tokyo Women's Medical University, Tokyo, Japan. ${ }^{2}$ Department of Medicine, Kidney Center, Tokyo Women's Medical University, 8-1 Kawada-cho, Shinjuku-ku, Tokyo 162-8666, Japan. ${ }^{3}$ Department of Nephrology, Jyoban Hospital, Fukushima, Japan.

Received: 8 July 2016 Accepted: 19 November 2016

Published online: 20 February 2017 


\section{References}

1. Nitta K, Tsuchiya K. Recent advances in the pathophysiology and management of protein-energy wasting in chronic kidney disease. Ren Replace Ther. 2016;2:4.

2. Fouque D, Kalantar-Zadeh K, Kopple J, Cano N, Chauveau P, Cuppari L, Franch H, Guarnieri G, Ikizler TA, Kaysen G, et al. A proposed nomenclature and diagnostic criteria for protein-energy wasting in acute and chronic kidney disease. Kidney Int. 2008;73:391-8.

3. Ikizler TA, Cano NJ, Franch H, Fouque D, Himmelfarb J, Kalantar-Zadeh K, Kuhlmann MK, Stenvinkel P, TerWee P, Teta D, et al. Prevention and treatment of protein energy wasting in chronic kidney disease patients: a consensus statement by the International Society of Renal Nutrition and Metabolism. Kidney Int. 2013;84:1096-107.

4. Kim JC, Kalantar-Zadeh K, Kopple JD. Frailty and protein-energy wasting in elderly patients with end stage kidney disease. J Am Soc Nephrol. 2013;24:337-51

5. Heimburger O, Qureshi AR, Blaner WS, Berglund L, Stenvinkel P. Hand-grip muscle strength, lean body mass, and plasma proteins as markers of nutritional status in patients with chronic renal failure close to start of dialysis therapy. Am J Kidney Dis. 2000;36:1213-25.

6. Isoyama N, Qureshi AR, Avesani CM, Lindholm B, Barany P, Heimburger O, Cederholm T, Stenvinkel P, Carrero JJ. Comparative associations of muscle mass and muscle strength with mortality in dialysis patients. Clin J Am Soc Nephrol. 2014;9:1720-8.

7. Johansen KL, Chertow GM, Jin C, Kutner NG. Significance of frailty among dialysis patients. J Am Soc Nephrol. 2007;18:2960-7.

8. Kalantar-Zadeh K, Kopple JD, Block G, Humphreys MH. A malnutritioninflammation score is correlated with morbidity and mortality in maintenance hemodialysis patients. Am J Kidney Dis. 2001;38:1251-63.

9. Steiber AL, Kalantar-Zadeh K, Secker D, McCarthy M, Sehgal A, McCann L. Subjective global assessment in chronic kidney disease: a review. J Ren Nutr. 2004;14:191-200.

10. Kalantar-Zadeh K, Kleiner M, Dunne E, Lee GH, Luft FC. A modified quantitative subjective global assessment of nutrition for dialysis patients. Nephrol Dial Transplant. 1999;14:1732-8.

11. Kobayashi I, Ishimura E, Kato Y, Okuno S, Yamamoto T, Yamakawa T, Mori K Inaba M, Nishizawa Y. Geriatric Nutritional Risk Index, a simplified nutritional screening index, is a significant predictor of mortality in chronic dialysis patients. Nephrol Dial Transplant. 2010;25:3361-5.

12. Yamada K, Furuya R, Takita T, Maruyama Y, Yamaguchi Y, Ohkawa S, Kumagai $H$. Simplified nutritional screening tools for patients on maintenance hemodialysis. Am J Clin Nutr. 2008;87:106-13.

13. Kalantar-Zadeh K, Kilpatrick RD, Kuwae N, McAllister CJ, Alcorn Jr H, Kopple JD, Greenland S. Revisiting mortality predictability of serum albumin in the dialysis population: time dependency, longitudinal changes and populationattributable fraction. Nephrol Dial Transplant. 2005;20:1880-8.

14. Friedman AN, Fadem SZ. Reassessment of albumin as a nutritional marker in kidney disease. J Am Soc Nephrol. 2010;21:223-30.

15. Masakane I, Nakai S, Ogata S, Kimata N, Hanafusa N, Hamano T, Wakai K, Wada A, Nitta K. An overview of regular dialysis treatment in Japan (as of 31 December 2013). Ther Apher Dial. 2015;19:540-74.

16. Kaysen GA, Dubin JA, Muller HG, Rosales L, Levin NW, Mitch WE, NIDDK HSG. Inflammation and reduced albumin synthesis associated with stable decline in serum albumin in hemodialysis patients. Kidney Int. 2004:65:1408-15.

17. Eustace JA, Coresh J, Kutchey C, Te PL, Gimenez LF, Scheel PJ, Walser M. Randomized double-blind trial of oral essential amino acids for dialysisassociated hypoalbuminemia. Kidney Int. 2000;57:2527-38.

18. Hiroshige K, Sonta T, Suda T, Kanegae K, Ohtani A. Oral supplementation of branched-chain amino acid improves nutritional status in elderly patients on chronic haemodialysis. Nephrol Dial Transplant. 2001;16:1856-62.

19. Kaysen GA, Greene T, Daugirdas JT, Kimmel PL, Schulman GW, Toto RD, Levin NW, Yan G, Group HS. Longitudinal and cross-sectional effects of Creactive protein, equilibrated normalized protein catabolic rate, and serum bicarbonate on creatinine and albumin levels in dialysis patients. Am J Kidney Dis. 2003;42:1200-11.

20. Nassar GM, Fishbane S, Ayus JC. Occult infection of old nonfunctioning arteriovenous grafts: a novel cause of erythropoietin resistance and chronic inflammation in hemodialysis patients. Kidney Int. 2002 61(Suppl 80): S49-54
21. Chen LP, Chiang CK, Chan CP, Hung KY, Huang CS. Does periodontitis reflect inflammation and malnutrition status in hemodialysis patients? Am J Kidney Dis. 2006;47:815-22.

22. Anders HJ, Andersen $\mathrm{K}$, Stecher B. The intestinal microbiota, a leaky gut, and abnormal immunity in kidney disease. Kidney Int. 2013;83:1010-6.

23. Girndt M, Sester M, Sester U, Kaul H, Kohler H. Molecular aspects of T- and B-cell function in uremia. Kidney Int Suppl. 2001;78:S206-211.

24. Rao M, Guo D, Perianayagam MC, Tighiouart $H$, Jaber BL, Pereira BJ, Balakrishnan VS. Plasma interleukin-6 predicts cardiovascular mortality in hemodialysis patients. Am J Kidney Dis. 2005;45:324-33.

25. Honda H, Qureshi AR, Heimburger O, Barany P, Wang K, Pecoits-Filho R, Stenvinkel P, Lindholm B. Serum albumin, C-reactive protein, interleukin 6, and fetuin a as predictors of malnutrition, cardiovascular disease, and mortality in patients with ESRD. Am J Kidney Dis. 2006:47:139-48.

26. Mehrotra R, Duong U, Jiwakanon S, Kovesdy CP, Moran J, Kopple JD, Kalantar-Zadeh K. Serum albumin as a predictor of mortality in peritoneal dialysis: comparisons with hemodialysis. Am J Kidney Dis. 2011:58:418-28.

27. Bergstrom J, Lindholm B. Malnutrition, cardiac disease, and mortality: an integrated point of view. Am J Kidney Dis. 1998;32:834-41.

28. Harnett JD, Foley RN, Kent GM, Barre PE, Murray D, Parfrey PS. Congestive heart failure in dialysis patients: prevalence, incidence, prognosis and risk factors. Kidney Int. 1995:47:884-90.

29. Foley RN, Parfrey PS, Harnett JD, Kent GM, Murray DC, Barre PE. Hypoalbuminemia, cardiac morbidity, and mortality in end-stage renal disease. J Am Soc Nephrol. 1996;7:728-36.

30. Ronco C, Haapio M, House AA, Anavekar N, Bellomo R. Cardiorenal syndrome. J Am Coll Cardiol. 2008;52:1527-39.

\section{Submit your next manuscript to BioMed Central and we will help you at every step:}

- We accept pre-submission inquiries

- Our selector tool helps you to find the most relevant journal

- We provide round the clock customer support

- Convenient online submission

- Thorough peer review

- Inclusion in PubMed and all major indexing services

- Maximum visibility for your research

Submit your manuscript at www.biomedcentral.com/submit
) Biomed Central 